\title{
Discussion on the Expression of Digital Model for the Residential Buildings of Han Style
}

\author{
Qianli Zhang \\ Macao University of Science and Technology, Hubei University, Hubei, Wuhan, 430062
}

Keywords: residential buildings of Han; digital; dynamic display

\begin{abstract}
This paper sums up the categories of buildings in Han dynasty and expounds characteristics of their buildings. It explains the digital significance of the construction of Chinese residential buildings. The dwelling houses building is discussed with several kinds of commonly used digital method: engineering drawing, the three dimensional mathematical model and dynamic display, and their respective features, and described based on the several ways to build the dwelling houses building model of the process; Then, this paper describes the virtual dynamic expression of dwelling houses building with several common display language: scripts, images, explanation, and music, by making dynamic demonstration of the dwelling houses building, explains how this display several language artistic processing and utilization, to achieve the purpose of optimization performance Han local-style dwelling houses building digital model; Finally, it puts forward a variety of intelligent terminal human-computer interaction platform performance and spreads Chinese local-style dwelling houses building digital model of the research results, to offer reference for the researchers enthusiastic on the dwelling houses building culture.
\end{abstract}

\section{Characteristics and Digital Significance of Han-style Residential Architecture}

\subsection{The Features of Han-style Residential Architecture.}

Wuhan, known as the "furnace", is a subtropical humid monsoon climate with abundant rainfall, abundant sunshine, numerous lakes, low terrain and abundant natural resources. While the ancestors thrived here, they wisely created their livable places and slowly formed the unique residential architecture style of Wuhan. These typical residential buildings have survived the wars of the past, and the "five parties" have spread throughout the three towns. For example, Wangzhai in Jiang'an District, Mao Zedong's former residence in Wuchang District, No. 81 Yanhualin in Wuchang District, former residence of Chen Changhao in Caidian District, Xuyuanquan Old Residence in Xinzhou District, Dayuwan Residential Quarter in Huanghua, Wenzwan Residential Group, and Wangjiaxiwan Residence Group and Luojiagang residential groups, and so on. These residential buildings have been weathered and historically precipitated, forming a residential style with unique living characteristics, collectively referred to as Han Style residential buildings.

The Han Style residential buildings are mostly in the form of a courtyard, with asymmetrical axes and a neat form. The courtyard consists of three main rooms, a two-story room and a patio. The front of the main house is a hall, the latter is a stove, and the left and the right are bedrooms. On the four sides of the patio, there are corridors connecting the gates, hatchbacks and main rooms. It is divided into one hospital, two hospitals and three hospitals. There is a separation belt between the courtyards as a pedestrian passage, called "Fire Lane". In order to adapt to the sultry weather and avoid the direct illumination of the sun, the depth of the residential buildings in Wuhan is generally large, which makes the indoors cool; the squatting is used to cover the sun; in order to prevent moisture, there are more attic storage items. The Han Style residential buildings generally use brick and wood structure. The façade is mostly in the three-open and five-open spaces. The outer wall uses a large area of clear water brick walls. It is also useful for stone. The stone is engraved with diagonal lines. Most of the Huanghua residences are made of local stone. "From the perspective of the building as a whole, there is a clear horizontal division of the feet, walls and eaves. The roof is mostly double-hill hard mountain type, but the shape of the steamed bread is 
various, because the requirements of ventilation and ventilation are high, and the roof is generally thinner than the northern area. Han Style residences inherit the traditional architectural style of the Ming and Qing Dynasties and are influenced by Western culture. For example, in the old residence of Xuyuanquan in Xinzhou District, the door pillars and gates are made of cement carvings with western architectural colors. Most of the residential buildings use a wooden frame that is worn by buckets, and there are also mixed types of buckets and beams. Due to the simple folk customs, it is rare to see the luxury decoration, mainly in the wall under the eaves, the gable end face and the threshold have a small number of folk stories painted. In addition, some wood carvings can be seen in the underarm bracing, the partition door, the window sill, etc. The patterns are fairy, bat, peony, etc., in order to be auspicious, these decorative patterns are not complicated but beautifully carved.

Wuhan, which was named "City Culture", was named "Historical and Cultural City" by the State Council in 1986. In recent years, Wuhan's urbanization process has kept pace with the times, and modern buildings are rapidly spreading throughout Jiangcheng. In 2017, Wuhan, which was named the "World Design Capital", once again entered the ranks of international cities. In order to let the Han-style dwelling architecture culture that carries Wuhan's historical and cultural heritage and slowly withdraw from people's sights spread at home and abroad, it is a far-reaching task to study how to digitally represent Han-style dwelling houses.

\subsection{The Digital Significance of Han-style Residential Architecture.}

In the digital age, through computer storage and processing, media information can be quickly promoted and disseminated in an open, compatible and shared manner. Digital technology has become the core and universal technology of all kinds of contemporary media. The digitization of residential buildings is to transform existing building information into data and form architectural digital models, including: flat drawing, three-dimensional models and dynamic virtual display. In the dynamic display of buildings, texts, images, voices, virtual reality, and information is integrated into various complex information through digital means, recreating the style of historical residential buildings, leaving precious visualizations for future generations. The Han-style residential buildings in the form of dynamic display, users can shuttle through the human-computer interaction platform in the residential buildings, and intuitively feel the architectural culture of Han-style dwellings. Therefore, the digital representation of Han Style residential architecture has its unique significance: 1. Facilitate the preservation, unified management and circulation of Han Style folk architecture and cultural information; 2. The digital dynamic display form is easy to present the historical features and humanistic features of Han Style residences, so that more Non-locals understand the residential buildings in Wuhan, attract foreign tourists to visit, promote the development of tourism in Wuhan, and spread the image of Wuhan city; 3. The digital restoration of Han Style residential buildings can evoke the historical memory of Wuhan people in the same era: use in the evening The well water sprinkled on the street to cool down, and the neighbors in the yard put a row of bamboo beds in front of the door to enjoy the coolness. The adults played cards and chatted together after dinner. The children played together and played scenes.....;4 digitized The combination of virtual reality technology can help realize virtual travel on the network and avoid the destruction of traditional residential buildings due to over-exploitation of tourism.

\section{The Digital Way of Han-style Residential Architecture}

The digitalization method of Han Style residential architecture is to combine computer graphics, image processing, computer vision and other disciplines with the discipline of architectural engineering, and apply the latest research results of the main digital-analog technology in the computer field to the digital representation of Han-style dwellings. Commonly used digital means include: engineering drawings, 3D digital simulation and dynamic display.

\subsection{Engineering Drawings.}

Get the dimensions before drawing the drawing. Field measurements, existing engineering drawings, 3D data point clouds, etc. can all capture the actual size of residential buildings. 
Generally, the Han Style residential building monolithic engineering plan includes the general plan of the building unit, the floor plan of each floor, the construction of the dimension table and other drawings, as shown in Figure 1, wherein each floor plan also includes the intersection of the building components, the door archway carvings and so on. The complete residential building engineering drawings usually include the diameter and height of the column roots of the civil construction; the length and height of various door arches; the main dimensions of the steps and thresholds; the size of the beams and rafts; the main dimensions of the doors and windows; The size of the hook and drip; the height and width of various ridges and the number of ridges of each slope of the tile roof.

Pay attention to the basic terms of the drawing when identifying drawings. For example, the type of the recognition line is divided into a solid line, a broken line, a single-dot long dash line, a double-dot chain line, and the like from the appearance of the line. The solid line can be divided into thick solid line, medium thick line and thin solid line. Usually, the thick solid line indicates the outer contour line of the building, and the middle thick line and thin solid line indicate the solid outline. Usually, it can also be represented by a thin solid line. Dimension lines, dimension lines, hatching lines, table border lines, etc., the thin dotted line indicates the axis, the symmetrical center line. Also identify architectural symbols such as cut-off symbols, detailed index symbols, symmetrical symbols, and so on. The types of dimensioning in architectural drawings are mainly length, diameter, radius, angle, and guide.

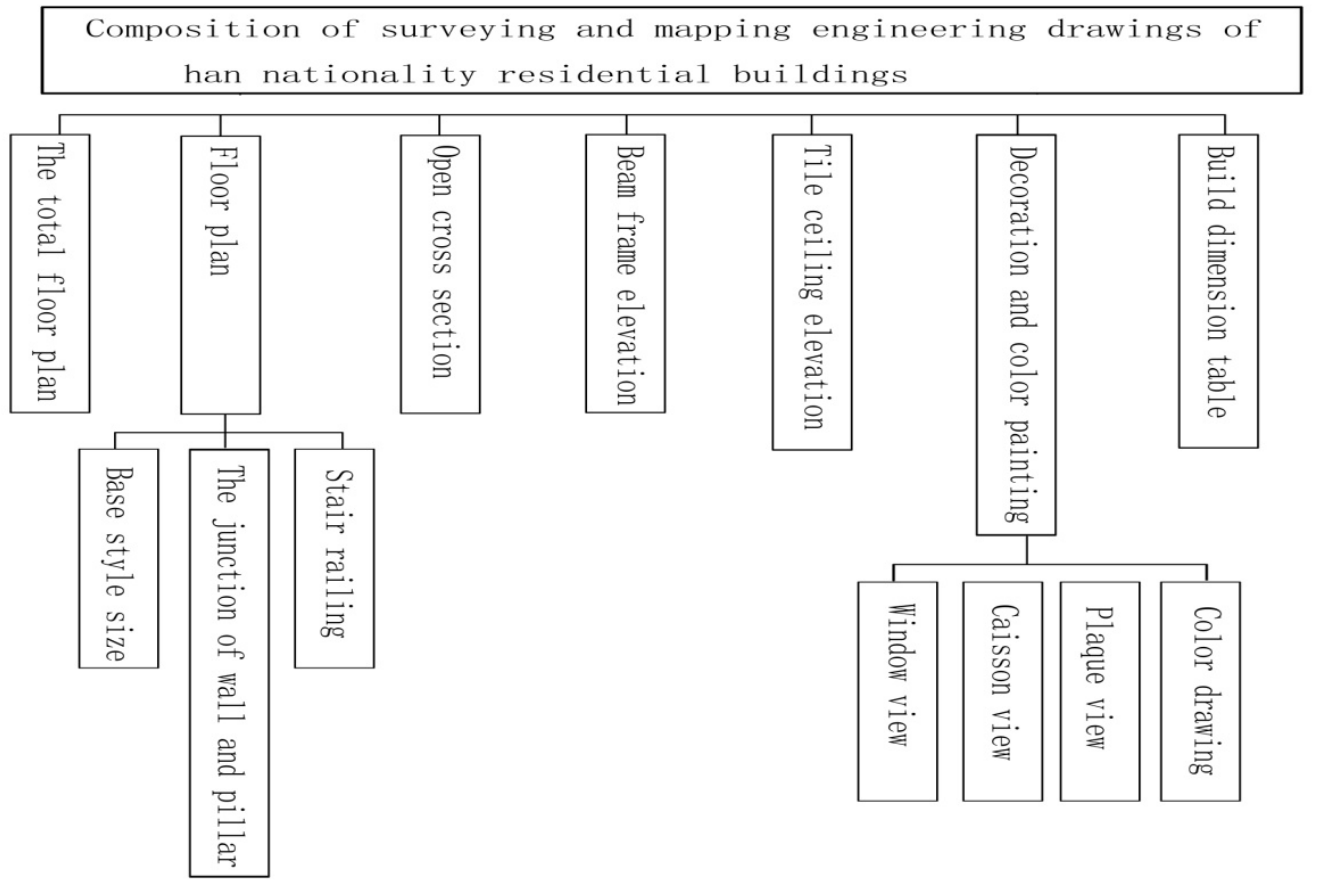

Fig.1

\subsection{Three-dimensional Digital Model.}

The three-dimensional digital model includes wireframe digital mode, surface digital mode and physical digital mode.

The wireframe digital model uses geometric ridges to represent the shape of the geometry. There is no surface, volume, etc. in the model. The wireframe model cannot generate surface texture, shadow, blanking, etc. It can be used as an initial digital construction method for Han Style residential buildings.

The surface digital model is a model of the outer surface structure of the geometric shape, just like a layer of skin on the wireframe digital pattern, so that the geometric shape has a certain contour, which can produce effects such as shadow and blanking. The surface digital model forms a 
three-dimensional digital surface from a two-dimensional object. The storage space for building graphics is small, the implementation speed is fast, and it is convenient to deal with texture mapping, shadow, blanking and the like. Some digital models, such as foundations, railings, beams, and slabs, have simple surface and regular geometric shapes, which are suitable for surface treatment. It is the main means of digital modeling construction of Han Style residential buildings.

The physical digital model is filled with a certain substance in the middle of the geometry, so that it has the characteristics of weight, density, etc., and can check the collision and interference of the two geometric bodies. The entity number model is based on simple entity digital model construction and editing, and adds processing methods for constructing and editing complex surface bodies. These complex and varied building entities include the connecting end and the occlusal end of different building components. These parts have many mouths and complex faces, and are easy to be applied by physical digital-analog means, but its data volume is large, and the amount of data contained is surface digital-analog. It is an auxiliary means of digital model construction of Han Style residential buildings.

Digital model construction of plane engineering drawings. Based on the recognition of the engineering drawing, the two-dimensional information is extracted for processing, and the three-dimensional building structure corresponding to the two-dimensional information is reconstructed. Usually based on the typical Han Pai residential survey paper, the three-dimensional digital model of residential buildings is constructed according to the residential building drawings, related dimensions and proportions.

Scanning the discretization information on the surface of the building through a non-contact 3D laser scanner, directly acquiring the façade data of the residential building, and then reducing the redundant information through reverse engineering, retaining the useful data point cloud, reconstructing and reconstructing This information corresponds to the exterior facade of the building. Obtaining data point cloud information setting sampling density should be reasonable and moderate, point cloud density is too large, will generate too much redundant information, if the density is too small, point cloud information can not reflect the details of the building entity. It is not affected by the complexity and light of the building to capture the surface data of the civil construction. It can obtain the three-dimensional digital model of the civil construction with strong realism and high-precision texture. It is helpful for the matching, reduction and reconstruction of digital and analog.

It is a method of reducing the architectural digital model by capturing image information of residential buildings from various angles by means of camera and the like. The advantage of image information over other means is that the texture pattern of the facade of the residential building can be extracted. After transformation, the corresponding surface of the three-dimensional digital model can be simulated to simulate the realism of the visual effect of the residential building.

\subsection{Dynamic Display.}

The digital dynamic virtual display of residential buildings refers to the continuous playback of multiple architectural scene images of a specified sequence within a valid time, thereby producing a regular and changing visual effect. Compared with the static display of residential buildings, it has great advantages. The dynamic display can be used to explore the entire building for multi-angle observation, using language explanations, and integrating the various atmospheres with the music to express the architectural effect. Not only that, but also the details of the building itself, the space between the buildings, and the layout of the landscape. The dynamic performance of the building dynamic virtual display is intuitive, comprehensive, in place, flexible and diverse. Intuitive dynamic presentations require artistic language for processing.

\section{Dynamic Display Language of Han-style Residential Building}

According to the author's experience in making architectural dynamic display cases, the digital dynamic display language of Han Style residential buildings can be summarized as: script, image, commentary and music. These artistic means are used to explore the production process and output 
of the digital virtual dynamic display of Han Style residential buildings.

\subsection{Script.}

Just like the script of film and television, "includes a description of all the environments in which the scene occurs, and a description of the actions that take place in those scenes." It stipulates the theme and positioning to be expressed in this dynamic display of the building. To a certain extent, the planner can predict what kind of psychological effects will be produced after the show is presented to the viewer. Although the script does not constitute a visual language for dynamic display, it is the soul that dominates the whole world.

\subsection{Image.}

The image quality used in the construction dynamic virtual display has high requirements. It includes both image resolution and size, as well as artistic processing of images. The specific refers to whether the image composition is appropriate, whether the color contrast and matching are appropriate, and whether the rendering effect is realistic. During the entire building demonstration process, the planner often adds several shots of the field to make the virtual display more realistic, which inevitably requires the virtual image to have realistic standards, otherwise the real image and the virtual image will be very different. For example, in the virtual display of the "Huangjiao Luojiagangwan Residential Group", in order to enhance the realism of the display, the author added the lens of the bridge shooting in the field, and the virtual building image around it is almost the same as the real scene effect. So the two "true and false" images are harmonious. If the image composition of the computer is unnatural, the color is unreasonable, and the rendering is not realistic, when playing these images, the viewer can easily visually distinguish the shot and the virtual lens in the field, which will affect the dynamic display effect.

Whether the image content contains delicate, vivid and close-up photos or close-ups is also an important factor affecting the level of dynamic display. In addition to showing the distant images of the large scenes of the building, there is also a close-up picture with rich details, which makes it more intriguing. If the viewer sees a close-up picture with realistic details and good artistic processing, it will naturally bring them some associations, which will be full of mystery and expectation to the surrounding architectural environment of the things in front of them. For example, the dynamic display of "Xuyuanquan's Old Residence in Xinzhou District" is an example of clever use of detailed close-up images, as shown in Figure 2. It is a virtual display film depicting the villa group. One of the reasons why this film can be highly praised is that there are more delicate and interesting close-up pictures in the film. As shown in the film: the white dove flying slowly in the sky, the green bamboo shaking in the breeze under the wall of the villa, the dwarf pine under the camphor tree, the shrubs filled with greenery on the stream, the blooming flowers in the green land, the grass The close-up pictures of flying butterflies and insects that permeate the eyes are vivid and vivid. There is also a slightly exaggerated and delicate art treatment, one of which is to slowly synthesize the indoor roaming scene into a crystal ball, followed by a close-up picture of the crystal ball close-up, the crystal clear crystal ball, the texture is crisp, Bright and seductive. Some architectural dynamic displays are often used in long-distance images. These images are viewed from a bird's eye view to create a holistic and magnificent atmosphere. In fact, this is not advisable. It will ignore the architectural details and the surrounding buildings. The details of the landscape and greenery lead to a dynamic display that gives a dull impression.

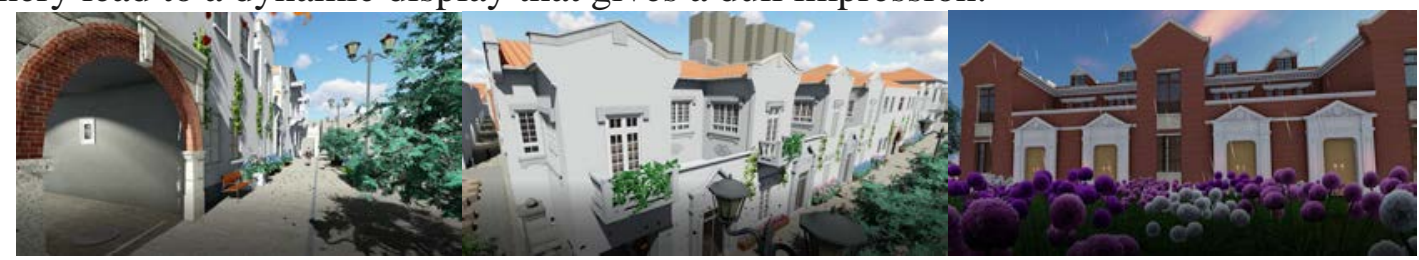

Picture A $\quad$ Picture B

Picture C

Figure 2 "Han Perry's Kun Qianli" clip picture

The fast and slow playback speed is also a factor that affects the display performance. 
Sometimes the image is played quickly to achieve the display. In the dynamic display of Shanghai Hongqiao Station, in order to reflect the fast and efficient theme of the station work, highlight the status and significance of the station, the entire display process has been using fast-paced playback speed, to meet the real life fast, the speed of the train, the economy The rapid development of a series of social significance brought by the station. In fact, this film has subtle artistic treatment in the detail control of the playback speed. For example, the speed of image playback in the first 40 seconds is extremely fast, the purpose is to attract the attention of the observer, to adjust the curiosity and passion that the audience expects; then, the station design idea emerges dynamically on the screen. Subtitle picture, usually this "dynamic emergence" playback effect is uninteresting, but after this kind of before and after playback speed comparison method, it becomes a novelty thing that the viewers are expecting. Sometimes the image playback speed must be slow. As mentioned above, the exhibition film "Xuyuanquan Old Residence in Xinzhou District", in order to reflect the beauty of the artistic concept of the villa scene, gives the viewer more appreciation of the aftertaste, showing a longer stay in the detail picture. Therefore, the playback speed of the image should be selected according to the theme of the display.

\subsection{Interpretation.}

Interpretation is also an indispensable component of dynamic display, and is the most direct expression of "language." Its role is to explain the various copywriting related to the building, including the geographical location of the building, the characteristics of the building, and the role of the building. Language selection should be stated. Proper explanations make the dynamic display of the building more memorable and impressive. For example, in the Shanghai Hongqiao Station conceptual design film, as a bidding design project, we see the characteristics of this design idea all the time. At first, it tells the overall shape of the station-the circle, with its own connotation and the use of the "circle" shape as the breakthrough point of the design idea, and then describes the functions of each part of the station. The film uses the vocal commentary language; some buildings use silent language, such as in the "Xuyuanquan Old Residence of Xinzhou District", in order to show the leisure and comfort of the villa group, highlight the appearance of the garden yashe, slow down in the picture The poems of two lines of characters are slowly appearing, and the poetic and artistic conception of the family style is vividly expressed by this silent and vocal language. In short, in the exhibition, we must play the artistic charm and effectiveness of the language.

\subsection{Music.}

Music is also a common expression technique. The artistic use of music can set off the atmosphere of the architectural scene. "In the use of music, it is generally necessary to consider the composition of the picture and the aesthetics of the music, and to take into account the music and the rhythm of the picture movement." In order to show the majestic momentum of the Hongqiao station building, with the fast-paced playback speed, the film is used in a hurry. In order to set off the secluded and antique charm of the celebrity family garden, the matching music selected in the film is full of artistic conception, and the sound of the guqin is immersive, and it is like immersing in the round-trip, as if entering the park. In short, the choice of music is very helpful for digital virtual display.

\section{Conclusion}

The Han Style residential architecture represents the architectural culture in a certain historical period. The unique architectural features contain a unique regional culture. It is an important part of Wuhan as a historical and cultural city. Through mature digital methods, such as plane engineering drawings, 3D digital simulation and virtual dynamic display, Han Style residential architecture, with professional virtual display language, with strong interactive design technology, spread Han Style residential architecture space and culture, to the current Scholars who study Han Style architecture and researchers who are interested in residential architecture are not a meaningful exploration. No matter what method is used to express the Hanpai architecture, it is not displayed in an archive after 
the event, but to promote them and bring out the value and significance of the digital results. Due to the many types of Han Style residential buildings and the complicated features, there is a vivid story behind each architectural detail. Therefore, the digital display promotion of Han Style residential buildings should be in line with the traditional one-way imported large-screen advertising form. Differently, it is necessary to use the current developed interactive technology to classify the digital results of Han Style residential buildings into a website-type human-computer interaction platform--a publicly-available APP service system or website that displays Han Style residential buildings in an orderly manner. The Han Style residential building corresponds to a link end, and the copywriting introduction combined with the dynamic virtual display can also introduce the building in detail. This kind of public service system APP should be compatible with smart terminals such as mobile phones, IPADs, PCs, etc., and spread on the WeChat or government websites, so that domestic and foreign enthusiasts of Hanpai folk culture can provide real-time overview of Hanpai residential buildings as a whole. The appearance can also carefully appreciate the architectural features and appreciate the customs behind it.

\section{Acknowledgement}

This article is the research result of the foundation item, which belongs to Hubei Intangible Cultural Heritage Protection Center in 2017(Hubei institute of fine art). The number of the project is FY-2017-03.

\section{References}

[1] Jeffrey Scott, Wang Yifu, Hou Yi, "Animation Script Creation and Marketing" [M]. Electronic Industry Press. 2000.6:74

[2] Ding Haixiang, Yao Guiping, editor. Introduction to Animation [M]. Tsinghua University Press. 2005.10:109

[3] Li Hesen. On the Artistic Expression Language of Product Virtual Dynamic Display[J]. Science and Technology Development. 2007(7): 47-48

[4] Guo Yacheng. The Changes and Development of Villages in Southeast Hubei Province_-Taking Yangxin, Tongshan and Chongyang County Villages as Examples [J]. Huazhong University of Science and Technology. 2007,6

[5] Li Baihao, Yang Jie. A preliminary study on Qi Neng, form and culture of Hubei native architecture [J]. Huazhong Architecture. 2007 (1): 176-179

[6] Zhang Zhenhua. Characteristics of Hubei Regional Culture and Han School Architecture [J]. Huazhong Architecture. 2009(1): 119-123

[7] Qin Wei.On the Formation and Development of Architectural Style in Hunan and Hubei Provinces[J].Huazhong University of Science and Technology.2003,5

[8] Wang Yansong. He Wei. Chinese Old Village - Yangxin Residence [J]. Hubei People's Publishing House. 2008

[9] Zhang Zuxun, Zhang Jianqing. Urban modeling approach and key technologies [J]. World Science and Technology Research and Development. 2003, 25(3): 23-29

[10] Tan Jianrong, Dong Yude. Size ring extraction algorithm based on graphical understanding and its implementation [J]. Computer research and development. 1999, 36(2): 192-196

[11] Zhang Shuyou, Peng Qunsheng, Tan Jianrong. Research on dimensionable labelability discrimination based on space base coordinates [J]. Computer journal. 2000, 23 (9): 982-986

[12] Li Kai, Li Li, Cai Shijie. A global identification method for semantic guidance in building structure diagrams [J]. Computer application. 2001, 21 (3): 40-43 
[13] Hu Wei, Yang Ruoyu, Cao Yang, etc. Three-dimensional reconstruction technology of building structure based on graphic understanding [J]. 2002, 13 (9): 1873 - 1880

[14] Bao Li, Lu Tong, Su Feng, etc. Research on 3D reconstruction technology of building structure based on information integration [J]. Journal of Computer-Aided Design \& Computer Graphics, Journal of Nanjing University (Natural Science Edition).2005.3,171-179

[15] Liu Hua, Hua Wei, Zhou Dong, etc. Semantic rules-driven Chinese ancient architectural modeling[J], Journal of Computer-Aided Design \& Computer Graphics.2004.16(10):1335-1340 